\title{
Use of blockchain technology in planning and management of transport systems
}

\author{
Luba Eremina ${ }^{1 *}$, Anton Mamoiko ${ }^{1}$ and $L i$ Bingzhang ${ }^{1}$ \\ ${ }^{1}$ Don State Technical University, Gagarina sq.1, Rostov on Don, 344000, Russia
}

\begin{abstract}
The paper presents the results of comparative studies of the transport management application blockchain technology. The accuracy of the use Quick Road System (QRS) in intelligent transport systems (ITS) may be the service of getting free passage is shown. This service is aimed at creation of decentralized network of road lane sharing in real time. Based on model studies it was found that, depending If a driver is in a hurry or wants to get priority in using the speed lane, then, having established a special status, he shares his place in the lane with other vehicles moving along the same route by exchanging incentives through the blockchain with other private car owners. The paper estimates the probability of with the development of Internet of Things (IoT) technology, the number of connected devices in ITS is growing extremely fast. Therefore, the optimal use of large arrays of collected data is the main focus of research and the Internet of Vehicles (IoV) is one of the most targeted branches of integration of the existing IoT technologies with the growing transport needs in order to solve the problem of intellectual traffic.
\end{abstract}

\section{Introduction}

Blockchain technology in planning and management of transport systems has great potential for use in the creation of self-sufficient and decentralized intelligent transport systems (ITS) [1]. Nevertheless, several key issues need to be addressed if the application of the cluster technology is to reach its full potential. The objective of the current study was to use block technology in the planning and management of transport systems in intelligent transport systems. With the development of Internet of Things (IoT) technology, the number of connected devices is growing extremely fast [2].

Therefore, the optimal use of large arrays of collected data is the main focus of research in various fields. Huge efforts have been made not only from an academic but also from an industrial point of view; the Internet of Vehicles (IoV) is one of the most targeted branches of integration of the existing IoT technologies with the growing transport needs in order to solve the problem of intellectual traffic. Road accidents can be as diverse and complex as serious traffic accidents or large congestion. However, preventative actions in the form of strict rules are far more effective than rapid 'ex post' responses. Therefore, the practical use of an Intelligent Transport System (ITS), which can indirectly improve traffic safety and

*Corresponding author: runa666.6@mail.ru 
reduce the number of accidents, is more than justified. A great deal of time and effort has been invested in empowering such ITS, which has brought countless benefits to society. At the same time, the benefits of such intelligent systems are always accompanied by potentially problematic security issues. This is where it makes sense to implement blockchain technology, i.e. the blockchain is expected to solve problems that may arise in a traditional centralized security system [3].

There are typically two important aspects to data protection: trust and privacy. Decentralized characterization of a blockaded system allows for a concept that proves the level of security. In this case, it is more reliable to share personal data only with users, and more data will be collected, leading to an intelligent monitoring system. The trust and anonymity of blockbusters also contributes to the concept that a person's personal rating can be closely linked to his or her behavior on the road. Consequently, a safer driver always enjoys a reputation as a reliable driver.

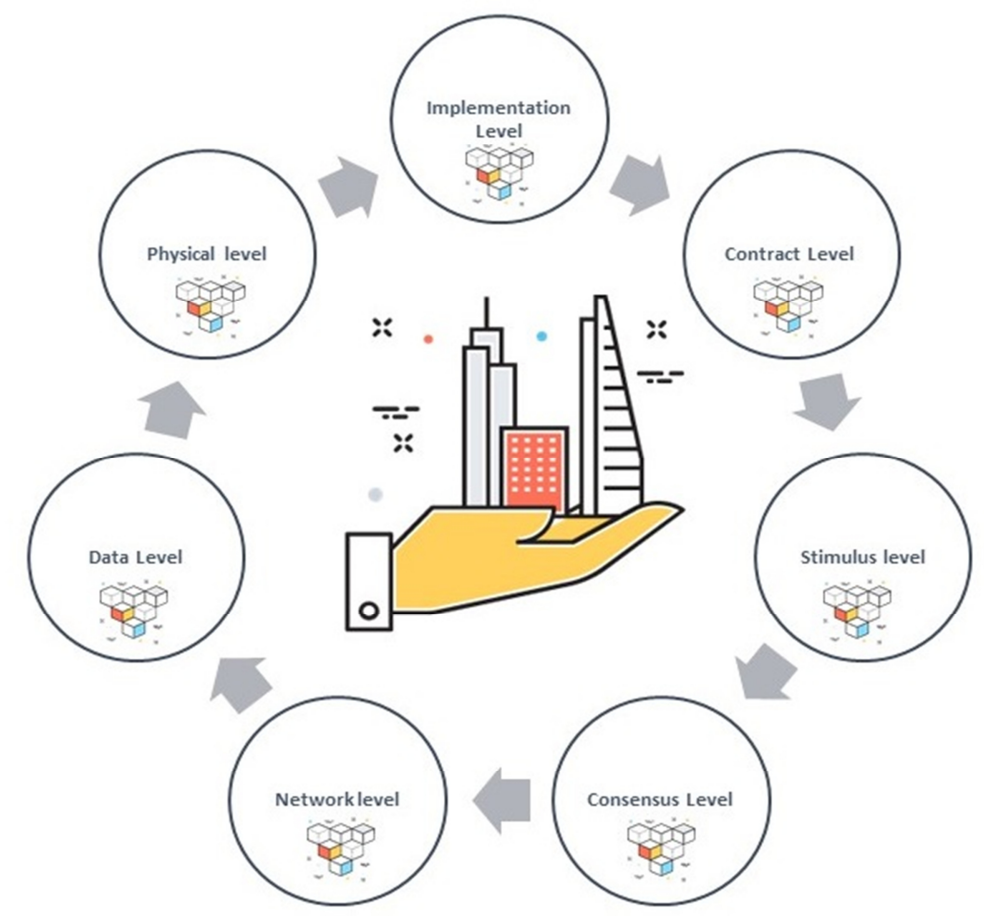

Fig. 1. Blockchain as a platform ITS

In order to make real-time monitoring and control of traffic more effectively coordinated, it is possible to link the individual rating to the road behaviour so that each person can benefit from safe driving. None of the rules of road traffic crashes can strictly force the creation of a more favourable road environment, but efforts should certainly be made by the public and the social environment. Consequently, through such monitoring and management of intelligent road traffic, accurate measures could be taken against those who are used to dangerous driving. In this way, it could improve the traffic situation during rush hours [4] .

In addition, a credit token, which is a blockbuster, could be used to record traffic violations by a specific driver. It is also possible to exchange "big data" between agencies, thus avoiding the isolation of ITS. It is possible to build a system that creates and integrates a credit token transaction based on blockades. Obviously, a credit token means a personal reputation, so in this case the credit scheme is valid only within a road platform. In order to 
help reduce accidents and create a more favourable transport environment, the token payment process is linked to both the correct lane change and the current choice of speed mode [5].

\section{Parallel transport management}

Let us define the fundamental research questions as basic capabilities and potential ideas.

1) Decentralized autonomous transport systems.

Decentralised autonomous transport systems include the main components of the block. P2P networks, based on consensus distributed coordination and economic benefits, are a natural way to model a complex transport system. Each computing node (for example, IoT devices, vehicles or other objects with computing power) can be considered as an autonomous agent in this system [6].

A large number of such nodes can be connected to a common network and communicate with each other through various types of block-based decentralized applications (Dapps), resulting in a decentralized autonomous organization (DAOs), subordinate to specific requirements and tasks. Next, when forming the macro-level, we will come to the creation of a decentralized autonomous system and even a community of systems (DAS) [7].

In this direction it is necessary to deepen into microlevel of individual behavior and interaction between autonomous agents of the system, as well as system macrolevel of modeling, self-organization, self-development and self-adaptation of systems.

2) Development of a mechanism to stimulate crowdsourcing.

The competition of the distributed consensus in the systems based on the block-chain technology can already be considered as a crowdsourcing task for a large number of nodes, which contribute their computing power to check the block-chain data [8]. These nodes are single agents, so the incentives and mechanisms of crowdsourcing should serve to ensure that the individual behavior of the node in its effort to maximize its income is coordinated with the system-wide goal of providing guarantees of protection and reliability of the system. The block technology used can be used to aggregate all possible computing resources in ITS to solve previously unsolvable problems, such as more precise real-time transportation management and control.

3) Software that determines the trust in its systems.

Confidence based on a chain of blocks in a system plays an important role in the creation of decentralized ITS, which in turn will allow to apply this technology to many tasks such as P2P commerce, payment and communication [9]. This type of trust is guaranteed by the code and verification of the majority of process participants. The technology has the potential to significantly reduce structurally complex systems, which in turn will reduce social problems. This will allow currency and assets to move freely between legal entities and individuals. For example, on the basis of P2P trust, the cars used can be resold and registered directly through the application blockchain instead of centralized bodies or platforms.

In this direction, issues that await further research are the fundamental rationale for building trust and trust management.

4) Intellectual contracts based on intelligent transport.

Smart contract serves as an "activator" of the blockchain, giving static data, using a variety of algorithms (for example, machine learning, analysis of large data, etc.) and highlevel programs of logic, to build the software ecosystem of ITS and improve the intelligence of its applications [10]. A self-executing intelligent contract significantly reduces social complexity by reducing the importance of the human factor, and can act as software agents on behalf of their creator or even themselves. Therefore, there is an urgent 
need to study the development and implementation of specific smart contracts and to manage and control ITS based on them.

5) Data security and privacy protection.

Although blockchain has shown high reliability and security, the encryption structure must be further strengthened in ITS with a large number of devices to protect against attacks.

A number of researchers [11] suggested the idea of PTMS (Parallel Transportation Management and Control System), which optimizes the real transport system by concurrently interacting with its respective artificial or virtual counterparts. Blockchain is one of the most secure and reliable architectures for PTMS, and thus, blockchain can be seen as an important step towards PTMS. One of the possible applications of blockchain technology in PTMS is shown in Figure 2 below.

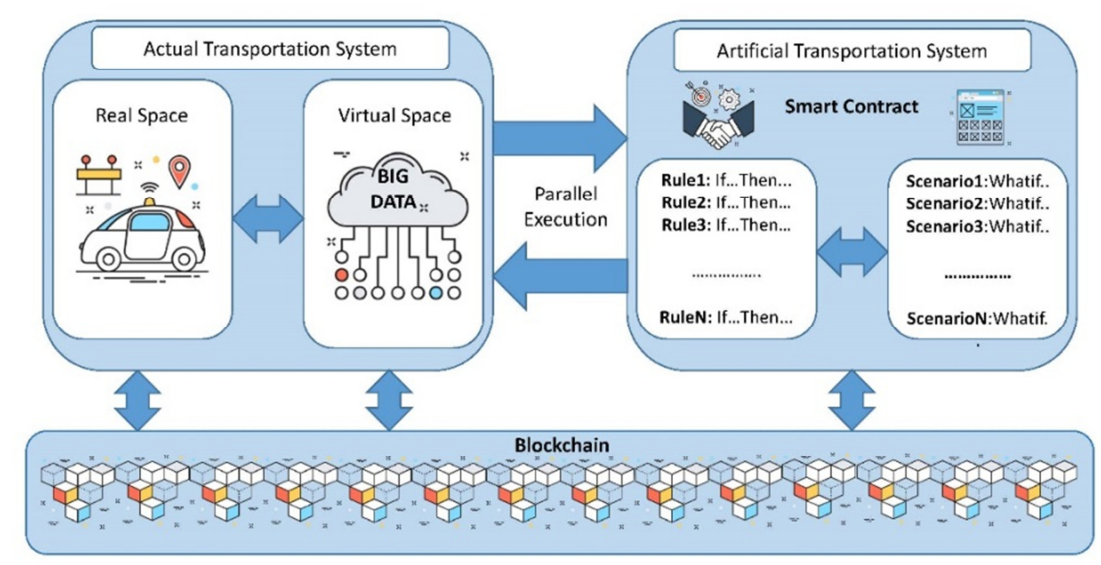

Fig. 2. Parallel transport management

A Blockchain-based PTMS will include all objects in physical space, including IoT devices, vehicles and assets, can be easily digitized through the "blockchain of things" and registered in the blockchain online. The transmission of large data in cyberspace can also be integrated into the blockchain. In addition, it is possible to create one or more artificial transport systems in the code space of smart contracts using the Ethereum platform, which offers programmable scripts to support complex modeling and computation [12].

Based on these jointly developed real and artificial transport systems, we are able to design and conduct multi-directional computational experiments to evaluate and validate specific behavior, mechanisms and strategies in ITS (e.g., to evaluate traffic conditions). These experiments can be designed as "What If" type of scenario output and modeling, based on predefined "If Then" rules. The optimal solution will be developed in a large number of computational experiments and will return to real transport systems. This process is repeated endlessly leading the actual transport system to the final approach of its optimal artificial analogues [13].

\section{Quick Road System}

One of the possible scenarios for the application of blockchecks in the ITS may be a service to obtain an unimpeded passage. Let us call it Quick Road System (QRS). This service is aimed at creating a decentralized network of road lane sharing in real time. If a driver is in a hurry or wants to get priority in using the speed lane, then, having established a special 
status, he shares his place in the lane with other vehicles moving along the same route by exchanging incentives through the blockchain with other private car owners.

A special application installed on the driver's smartphone or integrated into the vehicle software can be registered as one of the QRS computing nodes called "Road miners". Realtime data is verified and stored on a community supported P2P network through which all lane and payment sharing behaviour is coordinated and performed. The road miners are connected to the P2P network without any central authority.

As a consensus algorithm, an innovative consensus algorithm can be proposed, called "proof of traffic", which encourages road miners to drive with the QRS application running on their smartphones or onboard computers. In this way, road miners can contribute to the community by sharing their traffic data along the way and helping QRS to build a local network of lane social use. As an award, QRS automatically generates new tokens called "QRS" for road miners, and these tokens can be used to pay for travel and other transport services. The more they drive in slow lanes, the more QRS tokens they earn. Accordingly, those drivers who use fast lanes will have to pay for fast lane service from their own funds or from previously accumulated QRS tokens (Fig.3).

\section{Quick Road System}

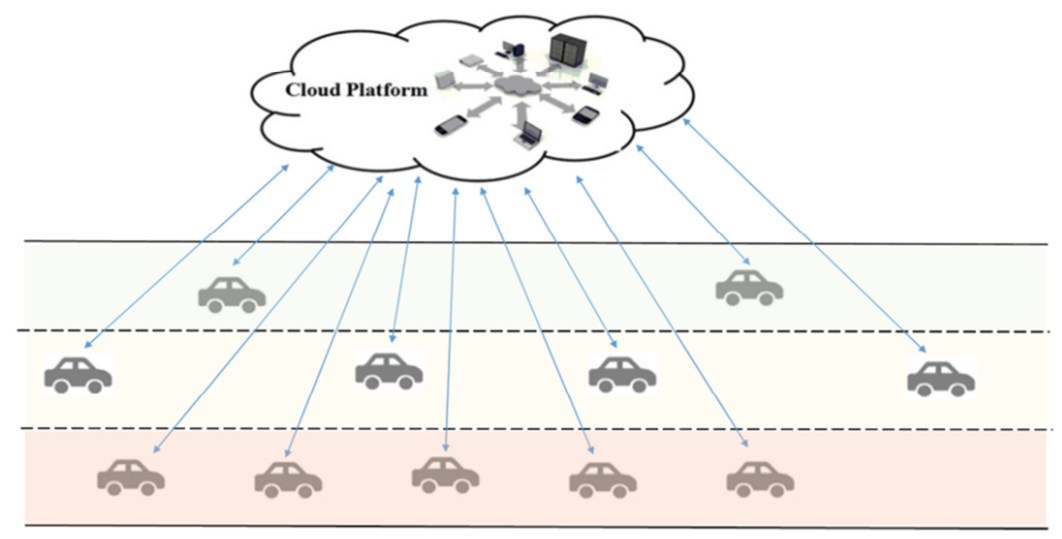

Fig. 3. The service of getting a free passage

While creating QRS, we will create various algorithms. These algorithms can be used to make specific decisions without human involvement, for example, to determine the possibility of using QRS in a particular geographic region or activate a service in a region where the number of active users exceeds the "critical mass", and so on.

The QRS will represent a decentralized, self-managed system (DAO) and together with other services, with a similar business model, represent a future trend of social transport and will change the economy of sharing [14-17].

Another variant of blockage application can be presented as a distributed transport service. Within a distributed network, own crypto coins are circulating. The network will be built on top of the Bitcoin Blockchain, and coins will be minted through a new concept proof of movement.

Anyone can join the transport network. To become a miner, you just need to download the application and turn on the GPS on your phone: the mining will start automatically when you move at a speed of over $20 \mathrm{~km} / \mathrm{h}$. You can also buy coins by participating in the development of the program code or design of the application, or just by attracting friends.

In this way, early supporters can lay the foundation for the future network until there are enough participants to run the entire system, where the drivers will be paid for with 
cryptocoins issued through the coin mechanism, for example, through Mastercoin, Counterparty or similar protocols [18].

It is only a matter of time before these two breakthrough concepts - distributed transportation and cryptovoltaics - are combined. We use crypt-currency technologies to gain the critical mass of users needed for smooth operation of the distributed transport network, as well as to create a truly distributed transport solution [19, 20].

The funds collected from coin purchases will be used for further development and improvement of the system. Purchased coins can be used to purchase trips in the future, when the network starts its real operation.

\section{Blockchain-based ITS security}

Mankind has learned to fly to and from the Moon, but at the same time on Earth we continue to stand in traffic jams. People are beginning to become very aware of the need to solve the transportation problem. Distributed transport services are a step in the right direction. But still only truly distributed systems can be fully trusted, because Uber and others like it are actually based on the old business formula. In contrast, block-chain systems are fully open, decentralized, belonging to a society where, in fact, anyone can become part of it $[21,22]$.

Blockchain-based ITS security issues

Infrastructure problems

Destroying equipment. The road infrastructure and the IMS are placed on the road, which means there is a risk of their destruction or hacking.

A network attack. There are two typical cases of network attacks:

- An external network attack. Distributed DDoS attacks, which try to send unwanted requests from different endpoints to disrupt the normal operation of the service network, is one of the typical attacks that typically occurs in a traditional centralized system.

- Internal data flow. With a large data stream it is likely that the system will experience pressure on transactions between hosts through the internal structure, which is seen as an internal overflow problem.

Protecting sensitive data

With an integrated blockage, three safety aspects are addressed:

1. Confidentiality. The data to be transferred can only be read by certain users designated by a "smart contract".

2. Honesty. Any data transaction will be recorded in a register, which receives and discloses the data recorded in it.

\section{Conclusions}

In the future, with the rapid development of artificial intelligence and $5 \mathrm{G}$ wireless connectivity, intelligent traffic will be formed into a comprehensive solution with a large number of IoT devices connecting and communicating with each other, so that traffic can be predicted and the optimal solution found in advance. In addition, a more intelligent system has been implemented, given the tremendous advances in intelligent software development.

It is expected that ITS integrated into the blockchain can become more powerful and efficient. This would help to reduce the influence of human factors and external malicious attacks in general, which would allow vehicles to communicate and self-organize according to traffic conditions through an intelligent contract in a chain of blocks.

In the future, it is expected that a machine learning algorithm will be developed to 
determine traffic intensity and predict traffic conditions, as well as a consensus block algorithm to increase tolerance and transaction rates in the real-time system.

The application of machine learning systems in the integrated process of data retrieval, loading and conversion is not only about the possibility of creating a more efficient system, but also about the possibility of creating a self-improving and self-managing service based on the ability of continuous learning. The analysed results of the collected sensory data and extensive data on the content of road modules will be processed and included in the knowledge base, so it is obvious that each subsequent quantitative analysis will be more accurate than the previous one. This indicates that road modules, which are mainly responsible for concentrating a large data stream, can adjust the data collection strategy to the results of the statistical analysis.

Blockchain technology is gradually emerging from its first deployments in cryptovoltaics and is now likely to have a significant impact in almost all industries. This technology is beginning to expand in all directions, including the logistics industry, where the Blockchain promises to improve the efficiency of business processes and facilitate the introduction of innovative services and business models.

\section{References}

1. J.-P. Zhang, F.-Y. Wang, K.-F. Wang, W.-H. Lin, X. Xu, C. Chen, IEEE Transactions on Intelligent Transportation Systems, 12, 1624-1639 (2011)

2. F.-Y. Wang, IEEE Transactions on Intelligent Transportation Systems, 11, 630-638 (2010)

3. L. Li, X. Li, Z. Li, D. Zeng, W.T. Scherer, IEEE Transactions on Intelligent Transportation Systems, 11, 251-255(2010)

4. M. Swan, Blockchain: Blueprint for a New Economy, (O'Reilly Media, Inc., 2015).

5. A. M. Antonopoulos, Mastering Bitcoin, (O’Reilly Media, Inc, 2014)

6. Y. Yuan, F.-Y. Wang. Acta Automatica Sinica, 42(4), 481-494 (2016)

7. Q.-J. Kong, L.-F. Li, B. Yan, S. Lin, F.-H. Zhu, G. Xiong, IEEE Intelligent Systems, 28, 66-69 (2013)

8. F.-Y. Wang, Control and Decision, 19(5), 485-489 (2004)

9. F.-Y. Wang, R. Dai, S. Zhang, G. Chen, S. Tang, D. Yang, X. Yang, and P. Li, Complex Systems and Complexity Science, 1(2), 60-69 (2004)

10. S. Nakamoto, Bitcoin: a peer-to-peer electronic cash system, online available: http://bitcoin.org/bitcoin.pdf, 2008

11. Blockchain technology and Internet of things, online available: http://www.newsbtc.com/2015/06/06/blockchain-technology-andinternet-of-things/, 2015

12. T.V. Lakshman, A.K. Agrawala, IEEE Transactions on Software Engineering, SE-12 600-607 (1986).

13. F. Kitahara, K. Kera, K. Bekki, Proceedings of International Workshop on Autonomous Decentralized Systems, 87-91 (2000)

14. K. Mori, High Integrity Software, 577, 89-111 (2001)

15. F.-Y. Wang, Complex Systems and Complexity Science, 1(4), 25-35 (2004)

16. Y.-S Lv, Y. OU, S.-M Tang, F.-H Zhu, H.-X Zhao, Journal of Jilin University (Engineeringand Technology Edition), 39, 87-90 (2009)

17. H.M. Kim, M. Laskowski, Intell. Syst. Account. Financ. Manag. 25, 18-27 (2018). 
18. M. Crosby, P. Pattanayak, S. Verma, V. Kalyanaraman, Appl. Innov. 2, 6-10 (2016).

19. H.R. Morley Industry Skeptical of Pace of Logistics Tech Adoption (JOC: New York, NY, USA, 2017).

20. W. Lehmacher Why Blockchain should be Global Trade's Next Port of Call; World Economic Forum: Geneva, Switzerland, 2017.

21. C.-S. Yang, T.-C. Lirn Int. J. Phys. Distrib. Logist. Manag. 47, 884-905 (2017) 